\title{
Prognostic markers and response to vandetanib therapy in sporadic medullary thyroid cancer patients
}

\author{
Vera Tiedje ${ }^{1, *}$, Saskia Ting2,*, Robert Fred Walter2,3, Thomas Herold2,4, Karl Worm², \\ Julia Badziong ${ }^{1}$, Denise Zwanziger ${ }^{1}$, Kurt Werner Schmid ${ }^{2}$ and Dagmar Führer ${ }^{1}$ \\ ${ }^{1}$ Department of Endocrinology and Metabolism, ${ }^{2}$ Institute of Pathology, ${ }^{3}$ Department of Interventional \\ Pneumology, Ruhrlandklinik, West German Lung Centre, University Hospital Essen, University of \\ Duisburg-Essen, Essen, Germany, and ${ }^{4}$ German Cancer Consortium (DKTK), German Cancer Research \\ Center (DKFZ), Heidelberg, Germany \\ *(V Tiedje and S Ting contributed equally to this work)
}

Correspondence should be addressed to $\mathrm{V}$ Tiedje Email

vera.tiedje@uk-essen.de

\begin{abstract}
Objective: Medullary thyroid carcinoma (MTC) occurs sporadically in 75\% of patients. Metastatic disease is associated with significantly poorer survival. The aim of this study was to identify prognostic markers for progressive MTC and oncogenic factors associated with response to vandetanib therapy.

Design and methods: Clinical courses of 32 patients with sporadic MTC ( $n=10$ pN0cM0, $n=8$ pN1cM0, $n=14$ pN1cM1) were compared with genetic profiles of the patients' primary tumour tissue. Analysis for RET proto-oncogene mutations was performed by Sanger sequencing and next-generation sequencing (NGS). The mRNA expression (mRNA count) of 33 targets was measured by nCounter Nanostring analysis.

Results: Somatic RET mutations occurred in 21/32 patients. The RET918 mutation was found in 8/14 pN1cM1 patients. $\operatorname{BRAF}(P=0.019), \operatorname{FGFR2}(P=0.007), \operatorname{FGFR3}(P=0.044)$ and VEGFC $(P=0.042)$ mRNA expression was significantly lower in $\mathrm{pN} 1 \mathrm{cM} 0 / \mathrm{pN} 1 \mathrm{cM} 1$ compared with pN0cM0 patients, whereas PDGFRA $(P=0.026)$ mRNA expression was significantly higher in pN1cM0/pN1cM1 when compared with pN0cM0 patients. Among the 10/32 vandetanib-treated patients, 5 showed partial response (PR), all harbouring the RET918 mutation. mRNA expression of FLT1 $(P=0.039), F L T 4$ $(P=0.025)$ and VEGFB $(P=0.042)$ was significantly higher in therapy responders.

Conclusions: In this study, we identified molecular markers in primary tumour tissue of sporadic MTC associated with the development of metastasis (both lymph node and organ metastasis) as well as response to vandetanib therapy.
\end{abstract}

\section{Introduction}

Medullary thyroid carcinoma (MTC) is a rare thyroid cancer and accounts for approximately 5\% of all thyroid malignancies. Curation is only possible by surgery. Advanced, metastasized disease results in poorer prognosis with a 10 -year survival of $40 \%$ or less (1). In approximately $25 \%$ of cases MTC occurs hereditary, due to a germline RET proto-oncogene mutation. In these patients with multiple endocrine neoplasia type 2 , a close correlation
() 2016 European Society of Endocrinology Printed in Great Britain is known between the type of RET mutation and onset of MTC (2). However, in 75\% of patients sporadic MTC is diagnosed. In these MTC somatic RET and RAS mutations, which occur mutually exclusive, are apparently the driver mutations $(3,4)$.

According to the American Thyroid Association (ATA) risk classification of RET germline mutations, RET918 mutations confer as the highest risk for early MTC 
manifestation and an aggressive clinical course. In sporadic MTC, the somatic RET918 mutation has been found as the most frequent RET mutation and has been linked to poorer survival $(5,6)$. Whether there is a correlation of other somatic RET mutations with distinct prognosis in sporadic MTC patients is currently not known (7).

In addition, activation of intracellular tyrosine kinase pathways has been linked to RET mutational status. Thus, Maliszewska et al. (8) reported upregulation of genes involved in the Wnt, Notch, NF-кB, JAK/STAT and MAPK signalling pathways in sporadic MTC harbouring the Met918Thr RET mutation. A RET-associated expression of tyrosine kinases was also described by Rodriguez-Antona et al. (9), whereby MTC with RET wildtype status showed a lower protein expression of PDGFRB than MTC with a somatic RET mutation.

However, whether somatic RET status and tyrosine kinase gene expression pattern are linked and associated with different disease course has not yet been elucidated.

The knowledge of the molecular pathology of MTC has led to development of therapies targeting RET and other tyrosine kinase signalling. Currently, two drugs are approved for treatment of progressive metastatic MTC, that is, vandetanib (European Medicines Agency (EMA) and Food and Drug Administration (FDA) approved), a RET, VEGFR2 (KDR), VEGFR3 (FLT4) and EGFR inhibitor (2) and cabozantinib (FDA approved, conditional EMA approved), a RET, VEGFR2, MET, KIT, AXL and FLT3 inhibitor $(2,10)$. A subanalysis with regard to progressionfree survival (PFS) in RET mutated vs RET wildtype patients was performed in a phase III trial and a benefit for PFS was suggested if a Met918Thr RET mutation was present in the tumour (2).

In this study, we determined RET mutation status by Sanger sequencing and next-generation sequencing (NGS) and performed quantitative expression analysis of a panel of 33 tumour-cell and endothelial-cell tyrosine kinases in sporadic MTC patients with long-term follow-up. Our aim was to delineate prognostic markers for metastasized MTC and to identify factors associated with response or absent response to vandetanib therapy.

\section{Methods}

\section{Patients and tumour samples}

In this study, 32 patients with sporadic MTC were included, all treated at the University Hospital Essen (Germany), a tertiary centre for endocrine malignancies. The study was approved by the Ethics Committee of the Medical Faculty, University Duisburg-Essen (Germany). Clinical data, including age at diagnosis, sex, TNM stage, postoperative calcitonin levels, and response to tyrosine kinase inhibitor (TKI) therapy, was analysed retrospectively. Ten patients received vandetanib therapy after tumour progress was radiologically demonstrated according to the Response Evaluation Criteria In Solid Tumors (RECIST) version 1.1. (11); response to therapy was classified as partial response $(\mathrm{PR})$, stable disease (SD), (12) or progressive disease (PD) 3 months after initiation of therapy. The time point 3 months was chosen to identify MTC patients with a primary vandetanib resistance.

Formalin-fixed paraffin-embedded (FFPE) tissues of the primary tumour were available in all cases of this study; diagnosis of MTC was reviewed by a board-certified, experienced thyroid pathologist (KWS). Hereditary MTC was excluded in all patients by germline RET gene mutation analysis from peripheral blood samples.

\section{Sanger sequencing of RET gene}

DNA was isolated from the primary tumour tissues with the QIAamp DNA FFPE tissue kit (Qiagen). For Sanger sequencing, RET exons 10, 11 and 13-16 were amplified by PCR. The PCR reaction was carried out in a total volume of $50 \mu \mathrm{L}$ with AmpliTaq Gold DNA Polymerase (Life Technologies), according to the recommendations of the supplier. Biometra T3000 Thermocycler (Biometra, Germany) was used for amplification. DNA sequences of both strands, forward and reverse, were analysed on an ABI 3500 Genetic Analyzer (Life Technologies), using BigDye Terminator chemistry (Life Technologies).

\section{Next-generation sequencing of RET gene}

For NGS, the MiSeq Illumina (Illumina, San Diego, CA, USA) was used following manufacturer's instructions. DNA concentrations were determined by Qubit 2.0 Fluorometer dsDNA HS assay kit (Life Technologies). Multiplex PCR and purification were performed with the GeneRead Gene Custom Panel, DNAseq Panel PCR Kit (Qiagen) and Agencourt AMPure XP Beads (Beckman Coulter, Brea, CA, USA), followed by measurement of total DNA amount by Qubit 2.0 Fluorometer dsDNA HS assay kit. The library preparation was performed with NEBNext Ultra DNA Library Prep Set for Illumina (New England Biolabs, Ipswich, MA, USA), according to the manufacturer's recommendations by using 24 different 
indices per run. The pooled library was sequenced on MiSeq (Illumina) and analysed by the Cancer Research Workbench (CLC Bio, Qiagen). For targeted sequencing, a customized MTC panel was designed containing regions of interest and hotspots of the RET gene. In all runs, an average coverage of approximately $8000 \times$ was obtained.

\section{Nanostring nCounter preparation and prestatistical analysis}

RNA was isolated from the primary tumour tissues with miRNAeasy FFPE kit (Qiagen) and total RNA concentrations were determined by using a fluorescent dye-based quantification method (Qubit, Life Technologies). Each sample was adjusted to a total RNA concentration of $200 \mathrm{ng} / \mu \mathrm{L}$ for the nCounter preparation following established protocols (13). For gene expression analysis, the General Purpose Reagent nCounter Elements TagSet- 48 and reagents according to the supplier were used (NanoString Technologies, Seattle, WA, USA). Probe Pools A ( $5 \mathrm{nM}$ per oligo) and B ( $25 \mathrm{nM}$ per oligo) were supplied by Integrated DNA Technologies (Coralville, IA, USA). The sample preparation was performed according to the nCounter Elements manual. The final hybridization was performed in an Eppendorf Mastercycler nexus (Hamburg, Germany) for $1.300 \mathrm{~min}$ at $67^{\circ} \mathrm{C}$ with a heated lid $\left(72^{\circ} \mathrm{C}\right)$. After hybridization, samples were directly loaded into the nCounter Prep Station (NanoString Technologies, Seattle, WA, USA) or cooled to $4^{\circ} \mathrm{C}$ until further proceeding within the next $4 \mathrm{~h}$. Samples were analysed on the nCounter Digital Analyzer (NanoString Technologies, Seattle, WA, USA) at 555 fields of view (FOV).

Table 1 Patient characteristics, somatic RET mutation status and ATA risk level.

\begin{tabular}{|c|c|c|c|c|c|c|c|}
\hline & $\underline{\text { Sex }}$ & Age at diagnosis & $\begin{array}{l}\text { TNM at the end of } \\
\text { follow-up }\end{array}$ & $\begin{array}{c}\text { Postoperative } \\
\text { calcitonin } \\
(\mathrm{pg} / \mathrm{mL}) \\
\end{array}$ & Sanger sequencing & $\begin{array}{l}\text { Next-generation } \\
\text { sequencing }\end{array}$ & Exon \\
\hline MTC1 & $\mathrm{F}$ & 58 & pT1pN0cM0 & $<2$ & c.2753T>C / p.Met918Thr & c. $2753 \mathrm{~T}>$ C / p.Met918Thr & Exon 16 \\
\hline MTC3 & M & 46 & pT2pN1cM1 & 7486 & c.1858T>C / p.Cys620Arg & c.1858T>C / p.Cys620Arg & Exon 10 \\
\hline MTC4 & $\mathrm{F}$ & 50 & pT3pN1cM0 & 64 & c.2753T>c / p.Met918Thr & c.2753T>C / p.Met918Thr & Exon 16 \\
\hline MTC5 & $\mathrm{F}$ & 29 & pT4pN1cM0 & $\mathrm{x}$ & c.2753T>C / p.Met918Thr & c. $2753 \mathrm{~T}>$ C / p.Met918Thr & Exon 16 \\
\hline MTC8 & M & 32 & pT2pN1bcM1 & 2363 & c.2753T>C / p.Met918Thr & c.2753T>C / p.Met918Thr & Exon 16 \\
\hline MTC12 & $\mathrm{F}$ & 45 & pT1pN0cM0 & $<2$ & c. $1858 \mathrm{~T}>\mathrm{A} /$ p.Cys620Ser & c. $1858 \mathrm{~T}>$ A / p.Cys620Ser & Exon 10 \\
\hline MTC13 & M & 53 & pT1bpN0cM0 & $<2$ & c. 1900 T>C / p.Cys634Arg & c. 1900 T>C / p.Cys634Arg & Exon 11 \\
\hline MTC14 & $\mathrm{M}$ & 58 & pT4pN1cM0 & 13 & & & \\
\hline MTC15 & M & 55 & pT3pN1cM0 & 830 & & & \\
\hline MTC16 & $\mathrm{F}$ & 56 & pT1pN1cM0 & 12 & c.2753T>c / p.Met918Thr & $\begin{array}{l}\text { c. } 2753 \text { T > C / p.Met918Thr } \\
\text { c. } 1900 T>\text { C / p.Cys634Arg } \\
\text { c. } 1946 \text { C > T / p.Ser649Leu }\end{array}$ & $\begin{array}{l}\text { Exon } 16 \\
\text { Exon } 11 \\
\text { Exon } 11\end{array}$ \\
\hline MTC18 & M & 55 & pT4pN1cM0 & 797 & c.2753T>C / p.Met918Thr & c. $2753 \mathrm{~T}>$ C / p.Met918Thr & Exon 16 \\
\hline MTC19 & $\mathrm{F}$ & 45 & pT3pN1cM0 & 31.866 & c.2753T>c / p.Met918Thr & c.2753T>c / p.Met918Thr & Exon 16 \\
\hline MTC21 & M & 44 & pT1pN1cM0 & $<2$ & $\begin{array}{l}\text { c. } 1900 \mathrm{~T}>\mathrm{C} / \text { p.Cys634Arg } \\
\text { c. } 1946 \mathrm{C}>\mathrm{T} / \text { p.Ser649Leu }\end{array}$ & $\begin{array}{l}\text { c. } 1900 T>C \text { / p.Cys634Arg } \\
\text { c. } 1946 \text { C > T / p.Ser649Leu }\end{array}$ & Exon 11 \\
\hline MTC23 & $\mathrm{F}$ & 43 & pT3pN1cM1 & 42 & & & \\
\hline MTC24 & $\mathrm{F}$ & 50 & pT3bpN1cM1 & 5 & c.2753T>C / p.Met918Thr & c.2753T>C / p.Met918Thr & Exon 16 \\
\hline MTC27 & M & 85 & pT2pN1cM0 & $<2$ & c.2753T>C / p.Met918Thr & c. $2753 \mathrm{~T}>$ C / p.Met918Thr & Exon 16 \\
\hline MTC31 & $\mathrm{F}$ & 39 & pT2pN0cM0 & $<2$ & & & \\
\hline MTC45 & M & 79 & pT1apN0cM0 & $<2$ & & & \\
\hline MTC48 & $\mathrm{F}$ & 75 & pT1pN0cM0 & $<2$ & & & \\
\hline MTC51 & $\mathrm{F}$ & 54 & pT1pN1cM1 & 135 & c.2753T>C / p.Met918Thr & c.2753T>C / p.Met918Thr & Exon 16 \\
\hline MTC54 & $\mathrm{F}$ & 64 & pT3pN0cM1 & 96 & & & \\
\hline MTC56 & M & 80 & pT2pN1bcM0 & 119 & c.2753T>C / p.Met918Thr & c.2753T>C / p.Met918Thr & Exon 16 \\
\hline MTC58 & $\mathrm{F}$ & 60 & pT1apN0cM0 & $<2$ & & & \\
\hline MTC63 & $\mathrm{F}$ & 49 & pT2apNOcM0 & $<2$ & & & \\
\hline MTC65 & $\mathrm{F}$ & 63 & pT1bpN1cM0 & 52 & c.2753T>C / p.Met918Thr & c. $2753 \mathrm{~T}>$ C / p.Met918Thr & Exon 16 \\
\hline MTC70 & $\mathrm{F}$ & 55 & pT1pN1cM0 & 13 & & & \\
\hline MTC71 & $\mathrm{F}$ & 72 & pT4pN1cM0 & 3 & c.2753T>c / p.Met918Thr & c.2753T>C / p.Met918Thr & Exon 16 \\
\hline MTC75 & $\mathrm{F}$ & 54 & pT3pN1bcM0 & 248 & c.2753T>C / p.Met918Thr & c.2753T>C / p.Met918Thr & Exon 16 \\
\hline MTC84 & $\mathrm{F}$ & 86 & pT3pN1acM0 & 1314 & & & \\
\hline MTC85 & $\mathrm{F}$ & 30 & pT1ap N1bcM0 & 7 & & & \\
\hline MTC91 & M & 65 & pT2pNOcM0 & $<2$ & c. $1888 \mathrm{~T}>$ C / p.Cys630Arg & c. $1888 \mathrm{~T}>$ C / p.Cys630Arg & Exon 11 \\
\hline MTC95 & $\mathrm{F}$ & 70 & pT4apN1bcM0 & 3 & & c.1902 C>G / Cys634Trp & Exon 11 \\
\hline
\end{tabular}


The raw data were analysed according to the following automated protocol: a background correction was performed, calculating the average counts for the negative controls plus two times the standard deviation. These values were subtracted from all sample counts. The geNorm algorithm (14) was used to identify the three most stable reference genes out of a set of nine reference genes (ACTB, GAPDH, HPRT1, GUSB, CLTC, NEDD8, PGK1, TTC1, TUBB). HPRT1, NEDD8 and TTC1 were identified to be the most stable reference genes. A normalization factor was calculated by using the geometric mean of the mRNA counts of the three most stable reference genes. Each sample was normalized using the sample-specific normalization factor. The normalized data were used for further statistical analysis (15).

\section{Statistical analysis}

Statistical analysis was performed by IBM SPSS Statistics Version 21. For comparison of mRNA count between MTC subgroups, Student's $t$-test was performed. Correlation of clinicopathological parameters with presence of sporadic RET mutations was calculated by Chi square. Statistical significance was set at a $P$-value $<0.05$.

\section{Results}

The clinical data of the 32 sporadic MTC included in this study are listed in Table 1 . The median age at diagnosis was 56 years. The patients were observed until December 2014 with a median observation period of 42 (3-159) months. Until then, 10 patients had disease-free status (pNOcM0), 8 patients had lymph node metastases (pN1cM0) and 14 patients developed distant metastatic disease (pN1cM1). Disease-free status was defined as biochemical and structural cure (calcitonin and CEA not detectable) during follow-up. Nine patients died of MTC during follow-up. Among the 32 patients, 14 received TKI treatment. Ten out of these 14 patients were started on vandetanib due to

Table 2 Characteristics of vandetanib-treated patients.

Sex

Female

Male

Age at diagnosis*

Lung

Lung + bone

Lung + bone + liver

Lung + bone + cerebral

Patients with PD under therapy

Median PFS, months

Deceased patients

Previous TKI treatment

Sorafenib

Cabozantinib
Characteristics

Site of metastasis

\begin{tabular}{c} 
Number \\
6 \\
4 \\
$58 \pm 10$ \\
2 \\
5 \\
2 \\
1 \\
7 \\
7 \\
6 \\
3 \\
2 \\
\hline
\end{tabular}

*Age in years (mean \pm s.D.). PD, progressive disease; PFS, progression-free survival.

PD according to RECIST criteria 1.1. Characteristics of the vandetanib-treated patients are listed in Table 2 . Among these 10 patients, 6 were treated as part of a clinical trial (ClinicalTrials.gov NCT01298323).

Sanger sequencing of the RET proto-oncogene revealed somatic RET mutations in the primary tumour tissues of 20 patients with sporadic MTC. The most frequent RET mutation was the Met918Thr mutation $(14 / 20,70 \%)$, followed by the Cys634Arg mutation (2/20, 10\%). Further somatic RET mutations, each found in only one case, included RET codons 620 (Cys620Arg and Cys620Ser), codon 630 (Cys630Arg) and codon 649 (Ser649Leu). Through NGS, one additional MTC with a RET mutations was found, which had not been detected by Sanger sequencing. Two further cases with somatic RET mutations already revealed by Sanger sequencing showed additional mutations in NGS. The detailed list of the somatic RET mutations identified in 32 sporadic MTCs is shown in Table 1.

The frequency of RET mutations in MTC in patients with metastatic ( $\mathrm{pN} 1 \mathrm{cM} 0$ and $\mathrm{pN} 1 \mathrm{cM} 1$ ) disease at the end of follow-up $(16 / 23,69.57 \%)$ differed from the frequency
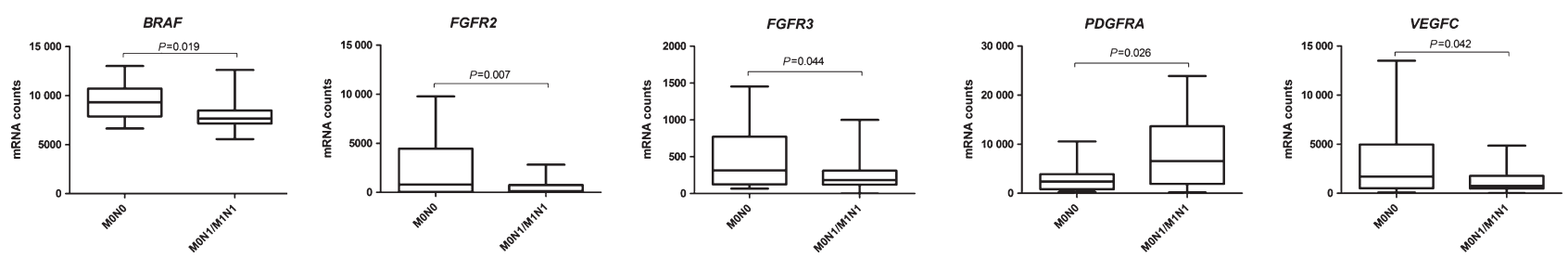

\section{Figure 1}

mRNA count in pNOcM0 vs pN1cM0/pN1cM1 patients. 

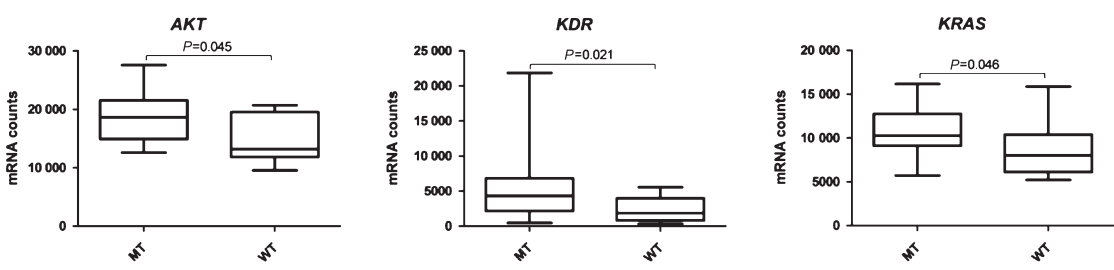

Figure 2

mRNA count in RET mutant vs RET wildtype MTC. in MTC of patients with pNOcM0-stage (5/10, 50.00\%, non significant $P=0.411$ ). The Met918Thr mutation was detected with significantly higher frequency in metastatic vs non-metastatic MTC ( $56.52 \%$ vs $10.00 \%, P=0.035)$. The Cys634Arg mutation was found in MTC of two patients with pNOcMO stage, who were cured by surgery and in two patients with metastatic disease.

Furthermore, we asked whether expression pattern of tyrosine kinases differed in tumours of patients with MTC confined to the thyroid (pNOcM0) vs patients showing or developing lymphonodular (pN1cM0) or distant metastatic disease (pN1cM1) at the end of follow-up. For this, quantitative nCounter analysis was employed and mRNA count for a panel of 33 distinct tumour-cell or endothelial-cell-derived tyrosine kinases was determined in the primary tumour tissues. We found that expression of BRAF $(P=0.019)$, FGFR2 $(P=0.007)$, FGFR3 $(P=0.044)$, PDGFRA $(P=0.026)$ and VEGFC $(P=0.042)$ differed significantly between MTC with pNOcMO stages and metastatic MTC (pN1cM0 and pN1cM1 stages) (Fig. 1). In case of metastatic disease, BRAF, FGFR2, FGFR3 and VEGFC were significantly downregulated, whereas expression of PDGFRA was significantly upregulated in the primary tumour tissues, in particular in patients with lymph node metastases $(P=0.007)$.

Comparison of RET mutation status with expression pattern of tyrosine kinases showed a proportional upregulation of $A K T 1(P=0.045), K R A S(P=0.046)$ and $K D R$ $(P=0.021)$ in MTC with somatic RET mutations vs RET wildtype status (Fig. 2). Especially MTC harbouring a RET Met918Thr mutation had significantly higher expression of AKT1 $(P=0.030)$, FGFR1 $(P=0.038), K D R \quad(P=0.015)$ and PDGFRA $(P=0.032)$ compared with MTC with RET wildtype status. Within the group of MTC harbouring somatic RET mutations, significant upregulation of FGFR1
$(P=0.027)$ and VEGFB $(P=0.049)$ was found in tumours with the RET Met918Thr mutation (data not shown).

Ten patients in our series were treated with vandetanib and were included in a subanalysis to determine whether RET mutation status and/or tyrosine kinase expression pattern in primary tumours of these patients were suitable markers to predict response. Response to vandetanib therapy was assessed at the initial staging after 3 months by RECIST 1.1 criteria. Five patients showed PR, three patients showed SD and two patients showed PD. All five patients showing PR had a MTC with a somatic Met918Thr RET mutation; however, in the small patient series we found no significant correlation between presence of a RET mutation in the primary tumour and treatment response at 3 months. In contrast, significantly higher expression of tyrosine kinases FLT1 $(P=0.039)$, FLT4 $(P=0.025)$ and $V E G F B(P=0.042)$ was found in MTC of patients showing PR to vandetanib, compared with patients with stable or $\mathrm{PD}$ at 3 months of vandetanib treatment (Fig. 3).

\section{Discussion}

In this study, somatic RET mutation status and expression pattern of tyrosine kinases were analysed in primary tumour tissues of 32 patients with sporadic MTC to determine whether genetic and/or signalling profiles could provide useful information on disease course and response to vandetanib treatment.

Parallel RET mutation analysis in MTC tissues by Sanger sequencing and NGS showed that the latter is more sensitive to detect somatic mutations in tumour tissues (4). In accordance with published literature, somatic RET mutations were detected in 70\% of sporadic MTC and Met918Thr was found as the most frequent somatic RET mutation in sporadic MTC (3).
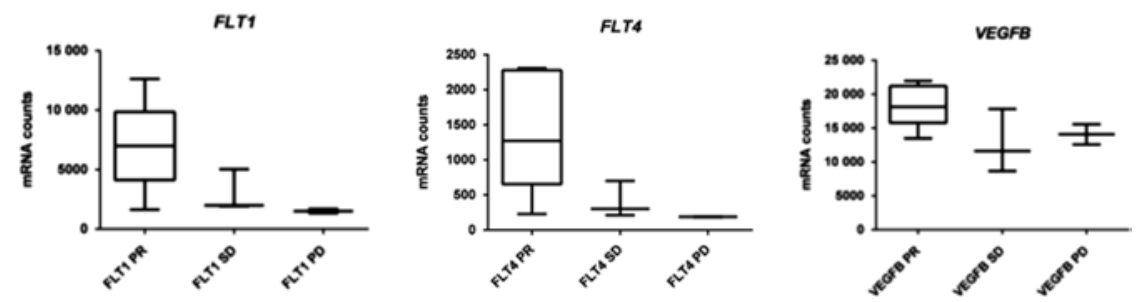

Figure 3

mRNA counts according to response to vandetanib therapy. Partial response (PR), stable disease (SD), progressive disease (PD). 
A risk stratification on the basis of the type of somatic RET mutation is so far less certain, mainly because of low patient numbers. In our series, the Met918Thr mutation was associated with distant metastatic disease in seven patients and this was a significant finding.

We further addressed the potential usefulness of tyrosine kinase expression pattern as prognostic markers in sporadic MTC. We found that the tumour-cell receptor tyrosine kinases FGFR2, FGFR3, the VEGFR ligand $V E G F C$ and the intracellular tyrosine kinase $B R A F$ were significantly downregulated in patients with metastatic MTC compared with patients cured by surgery. In contrast, PDGFRA, located at endothelial cells (e.g., blood vessels), was significantly upregulated in metastatic MTC. Previously reported increase of EGFR protein expression in patients with metastatic disease (16) could not be confirmed in our study (data not shown).

The fibroblast growth factor receptor (FGFR) family is essential for proliferation, survival and angiogenesis (17). Its role in cancer has been studied broadly in gastric (18), endometrial (19) and lung cancer (20) and FGFR gene mutations, amplifications and overexpression have been described. Bernard et al. (21) investigated the expression of FGFR1-4 in normal thyroid tissue as well as in papillary, follicular and poorly differentiated thyroid cancer. Interestingly, FGFR2 was the only receptor to be expressed in normal thyroid tissue and was downregulated in thyroid carcinoma, suggesting a protective role of FGFR2 in carcinogenesis. This is in accordance with our results, showing a higher FGFR2 mRNA count in patients with localized vs metastatic MTC.

Since the MAPK pathway is activated through the RET receptor tyrosine kinase in MTC (22), expressions of $B R A F$ and the downstream mitogen-activating kinases (MAP2K1, MAP2K2, MAPK1, MAPK3) were investigated in our study. Interestingly, the mRNA count of $B R A F$ is significantly higher in MTC of patients with disease-free status cured after surgery in comparison to tumours of patients with metastatic disease. In contrast, the mRNA counts of the mitogen-activating kinases are similar in both subgroups (data not shown). The mechanism of MAPK activation differs in follicular-cell-derived tumours from medullary thyroid cancer. In follicularcell-derived tumours, for example, papillary thyroid cancer, BRAF mutations are often causative (23). In medullary thyroid cancer, MAPK activation is effectuated through the RET mutations (22). The higher BRAF count in cured patient could imply that BRAF is involved in the initial tumour development, but is not essential for tumour progression.
VEGF are specific ligands of the VEGFR family and are secreted by tumour cells, promoting lymph angiogenesis, blood vessel growth and tumour progression (24). In our study, we found that the VEGFC mRNA count is significantly higher in primary tumours of non-metastatic MTC compared with metastatic MTC disease, while VEGFA and $V E G F B$ expression is similar (data not shown). VEGFC is a KDR (VEGFR2) and FLT1 (VEGFR3) ligand. KDR and FLT1 mRNA counts show an opposite expression pattern with KDR upregulation and FLT1 downregulation in the primary tumour tissue of patients with metastatic disease.

Rodriguez-Antona et al. (16) reported a higher protein expression of KDR in metastatic tissues compared with primary tumour tissue of MTC patients. Our data suggest that a higher KDR mRNA count in MTC mirrors a more aggressive tumour biology, while high VEGFC and FLT1 expression are compatible with disease-free status at the end of follow-up.

Platelet-derived growth factor receptors are a family of receptor tyrosine kinases located at the membrane of endothelial cells and are promoting cell survival, growth and proliferation. The overexpression of PDGFRs has been demonstrated in various cancers, such as prostate (25), ovarian (26) and non-small-cell lung cancer (27). In medullary thyroid cancer, this has so far not been investigated. Zhang et al. (28) analysed PDGFRA expression in normal thyroid and papillary thyroid cancer tissue. PDGFRA expression was significantly higher in thyroid cancer tissues, especially in PTC with lymph node metastases. In our MTC series, we also found that the PDGFRA mRNA count was significantly higher in MTC with lymph node or distant metastases in comparison to MTC with NOMO stage. Therefore, it can be hypothesized that the PDGFRA pathway might play a role in the development of lymphatic and haematogenous metastases. Targeting the PDGFRA pathway is a potential therapeutic approach, and sorafenib, sunitinib and pazopanib are also inhibitors of this tyrosine kinase receptor. For sorafenib (29), sunitinib (30) and pazopanib, (31) phase II trials have shown some treatment response in medullary thyroid cancer. However, so far no phase III studies have been performed.

In this study, we also addressed response markers to vandetanib treatment. First, among the five MTC patients showing PR, all had a tumour with a somatic RET918 mutation. Second, the FLT1, FLT4 and FLT1 ligand VEGFB mRNA expression were significantly higher in vandetanib responders. If these findings can be reproduced in larger patient series, this may suggest the potential for a molecular stratification on the basis of primary 
tumour tissue profiling to predict vandetanib response in metastatic MTC. RET mutations located in codon 804 and 806 are known to cause in vitro resistance to vandetanib therapy $(32,33)$. In our cohort, no mutations in codon 804 or 806 were detected.

The limitations of this study are its retrospective nature and the limited patient number. Furthermore, we are aware of a potential bias through negative selection of patients that had developed metastases during follow-up. Thus, due to the storage limitation of FFPE in non-university facilities (no longer than 10 years), many MTC patients with pNOcM0 stages were excluded from our study because patients were cured and no tissue was available. Thus except one, the patients in our study had distant metastatic disease diagnosed less than 10 years after initial MTC diagnosis, representing a more aggressive tumour behaviour. However, our aim was to identify molecular markers that could serve as prognostic factors indicating a more benign or aggressive nature of sporadic MTC. We think that such a comparison between our patients group is valid and that this approach is useful to generate marker profiles that can be evaluated and validated in a multicentre approach involving larger patient series.

\section{Conclusion}

In this study, we have reported for the first time that expression pattern of tumour-cell receptor tyrosine kinases and endothelial receptor tyrosine kinases in the primary tumour tissues of sporadic medullary thyroid cancer are linked with tumour stage and development of metastases during follow-up. Moreover, the presence of a somatic RET Met918Thr mutation and higher PDGFRA and $K D R$ expression indicates a more aggressive tumour type with increased risk for metastatic disease. As regards targeted therapy, we show for the first time that the somatic RET918 mutation and the expression of FLT1, FLT3 and VEGFB in primary tumour tissues are linked with PR to vandetanib.

\section{Declaration of interest}

$D F$ and $V T$ have received speaker fees and have served on advisory boards for AstraZeneca. S T, R F W, T H, K W, J B, D Z and K W S have no conflict of interest.

\section{Funding}

This research did not receive any specific grant from any funding agency in the public, commercial or not-for-profit sector.

\section{Acknowledgements}

We thank the MOLBIZ - Molekular Biologisches Zentrum Ruhrlandklinik, University Hospital Essen, University of Duisburg-Essen, Germany for performing the nCounter preparation and data generation.

\section{References}

1 Roman S, Lin R \& Sosa JA. Prognosis of medullary thyroid carcinoma: demographic, clinical, and pathologic predictors of survival in 1252 cases. Cancer 2006107 2134-2142. (doi:10.1002/(ISSN)1097-0142)

2 Wells SA Jr, Robinson BG, Gagel RF, Dralle H, Fagin JA, Santoro M, Baudin E, Elisei R, Jarzab B, Vasselli JR et al. Vandetanib in patients with locally advanced or metastatic medullary thyroid cancer: a randomized, double-blind phase III trial. Journal of Clinical Oncology 201230 134-141. (doi:10.1200/JCO.2011.35.5040)

3 Agrawal N, Jiao Y, Sausen M, Leary R, Bettegowda C, Roberts NJ, Bhan S, Ho AS, Khan Z, Bishop J et al. Exomic sequencing of medullary thyroid cancer reveals dominant and mutually exclusive oncogenic mutations in RET and RAS. Journal of Clinical Endocrinology and Metabolism 201398 E364-E369. (doi:10.1210/ jc.2012-2703)

4 Simbolo M, Mian C, Barollo S, Fassan M, Mafficini A, Neves D, Scardoni M, Pennelli G, Rugge M, Pelizzo MR et al. High-throughput mutation profiling improves diagnostic stratification of sporadic medullary thyroid carcinomas. Virchows Archiv 2014465 73-78. (doi:10.1007/s00428-014-1589-3)

5 Schilling T, Burck J, Sinn HP, Clemens A, Otto HF, Hoppner W, Herfarth C, Ziegler R, Schwab M \& Raue F. Prognostic value of codon 918 (ATG-->ACG) RET proto-oncogene mutations in sporadic medullary thyroid carcinoma. International Journal of Cancer 200195 62-66. (doi:10.1002/(ISSN)1097-0215)

6 Elisei R, Cosci B, Romei C, Bottici V, Renzini G, Molinaro E, Agate L, Vivaldi A, Faviana P, Basolo F et al. Prognostic significance of somatic RET oncogene mutations in sporadic medullary thyroid cancer: a 10-year follow-up study. Journal of Clinical Endocrinology and Metabolism 200893 682-687. (doi:10.1210/jc.2007-1714)

7 Moura MM, Cavaco BM, Pinto AE, Domingues R, Santos JR, Cid MO, Bugalho MJ \& Leite V. Correlation of RET somatic mutations with clinicopathological features in sporadic medullary thyroid carcinomas. British Journal of Cancer 2009100 1777-1783. (doi:10.1038/sj.bjc.6605056)

8 Maliszewska A, Leandro-Garcia LJ, Castelblanco E, Macia A, de Cubas A, Gomez-Lopez G, Inglada-Perez L, Alvarez-Escola C, De la Vega L, Leton R et al. Differential gene expression of medullary thyroid carcinoma reveals specific markers associated with genetic conditions. American Journal of Pathology 2013182 350-362. (doi:10.1016/j.ajpath.2012.10.025)

9 Rodriguez-Antona C, Munoz-Repeto I, Inglada-Perez L, de Cubas AA, Mancikova V, Canamero M, Maliszewska A, Gomez A, Leton R, Leandro-Garcia LJ et al. Influence of RET mutations on the expression of tyrosine kinases in medullary thyroid carcinoma. Endocrine-Related Cancer 201320 611-619. (doi:10.1530/ERC-12-0316)

10 Elisei R, Schlumberger MJ, Muller SP, Schoffski P, Brose MS, Shah MH, Licitra L, Jarzab B, Medvedev V, Kreissl MC et al. Cabozantinib in progressive medullary thyroid cancer. Journal of Clinical Oncology 2013 31 3639-3646. (doi:10.1200/JCO.2012.48.4659)

11 Eisenhauer EA, Therasse P, Bogaerts J, Schwartz LH, Sargent D, Ford R, Dancey J, Arbuck S, Gwyther S, Mooney M et al. New response evaluation criteria in solid tumours: revised RECIST guideline (version 1.1). European Journal of Cancer 200945 228-247. (doi:10.1016/j.ejca.2008.10.026)

12 Mulligan LM, Kwok JB, Healey CS, Elsdon MJ, Eng C, Gardner E, Love DR, Mole SE, Moore JK, Papi L et al. Germ-line mutations of the RET proto-oncogene in multiple endocrine neoplasia type 2A. Nature 1993363 458-460. (doi:10.1038/363458a0) 
13 Walter RF, Mairinger FD, Werner R, Ting S, Vollbrecht C, Theegarten D, Christoph DC, Zarogoulidis K, Kurt Werner S, Zarogoulidis P et al. SOX4, SOX11 and PAX6 mRNA expression was identified as a (prognostic) marker for the aggressiveness of neuroendocrine tumours of the lung by using next-generation expression analysis (NanoString). Future Oncology 201511 1027-1036. (doi:10.2217/fon.15.18)

14 Hruz T, Wyss M, Docquier M, Pfaffl MW, Masanetz S, Borghi L, Verbrugghe P, Kalaydjieva L, Bleuler S, Laule O et al. RefGenes: identification of reliable and condition specific reference genes for RT-qPCR data normalization. BMC Genomics 201112156. (doi:10.1186/1471-2164-12-156)

15 Mairinger FD, Walter RF, Werner R, Christoph DC, Ting S, Vollbrecht C, Zarogoulidis K, Huang H, Li Q, Schmid KW et al. Activation of angiogenesis differs strongly between pulmonary carcinoids and neuroendocrine carinomas and is crucial for carcinoid tumourgenesis. Journal of Cancer 20145 465-471. (doi:10.7150/jca.9235)

16 Rodriguez-Antona C, Pallares J, Montero-Conde C, Inglada-Perez L, Castelblanco E, Landa I, Leskela S, Leandro-Garcia LJ, LopezJimenez E, Leton R et al. Overexpression and activation of EGFR and VEGFR2 in medullary thyroid carcinomas is related to metastasis. Endocrine-Related Cancer 201017 7-16. (doi:10.1677/ERC-08-0304)

17 Katoh M. Cancer genomics and genetics of FGFR2 (review). International Journal of Oncology 200833 233-237.

18 Jang JH, Shin KH \& Park JG. Mutations in fibroblast growth factor receptor 2 and fibroblast growth factor receptor 3 genes associated with human gastric and colorectal cancers. Cancer Research 200161 3541-3543.

19 Pollock PM, Gartside MG, Dejeza LC, Powell MA, Mallon MA, Davies H, Mohammadi M, Futreal PA, Stratton MR, Trent JM et al. Frequent activating FGFR2 mutations in endometrial carcinomas parallel germline mutations associated with craniosynostosis and skeletal dysplasia syndromes. Oncogene 200726 7158-7162. (doi:10.1038/sj.onc.1210529)

20 Davies H, Hunter C, Smith R, Stephens P, Greenman C, Bignell G, Teague J, Butler A, Edkins S, Stevens C et al. Somatic mutations of the protein kinase gene family in human lung cancer. Cancer Research 200565 7591-7595.

21 St Bernard R, Zheng L, Liu W, Winer D, Asa SL \& Ezzat S. Fibroblast growth factor receptors as molecular targets in thyroid carcinoma. Endocrinology 2005146 1145-1153.

22 Drosten M \& Putzer BM. Mechanisms of disease: cancer targeting and the impact of oncogenic RET for medullary thyroid carcinoma therapy. Nature Clinical Practice Oncology 20063 564-574. (doi:10.1038/ncponc0610)

23 Romitti M, Ceolin L, Siqueira DR, Ferreira CV, Wajner SM \& Maia AL. Signaling pathways in follicular cell-derived thyroid carcinomas (review). International Journal of Oncology 201342 19-28. (doi:10.3892/ijo.2012.1681)

24 Achen MG \& Stacker SA. Tumor lymphangiogenesis and metastatic spread-new players begin to emerge. International Journal of Cancer 2006119 1755-1760.

25 Chott A, Sun Z, Morganstern D, Pan J, Li T, Susani M, Mosberger I, Upton MP, Bubley GJ \& Balk SP. Tyrosine kinases expressed in vivo by human prostate cancer bone marrow metastases and loss of the type 1 insulin-like growth factor receptor. American Journal of Pathology 1999155 1271-1279. (doi:10.1016/S0002-9440(10)65229-7)

26 Henriksen R, Funa K, Wilander E, Backstrom T, Ridderheim M \& Oberg K. Expression and prognostic significance of platelet-derived growth factor and its receptors in epithelial ovarian neoplasms. Cancer Research 199353 4550-4554.

27 Donnem T, Al-Saad S, Al-Shibli K, Andersen S, Busund LT \& Bremnes RM. Prognostic impact of platelet-derived growth factors in non-small cell lung cancer tumor and stromal cells. Journal of Thoracic Oncology 20083 963-970. (doi:10.1097/JTO.0b013e3181834f52)

28 Zhang J, Wang P, Dykstra M, Gelebart P, Williams D, Ingham R, Adewuyi EE, Lai R \& McMullen T. Platelet-derived growth factor receptoralpha promotes lymphatic metastases in papillary thyroid cancer. Journal of Pathology 2012228 241-250. (doi:10.1002/path.v228.2)

29 Lam ET, Ringel MD, Kloos RT, Prior TW, Knopp MV, Liang J, Sammet S, Hall NC, Wakely PE Jr, Vasko VV et al. Phase II clinical trial of sorafenib in metastatic medullary thyroid cancer. Journal of Clinical Oncology 201028 2323-2330. (doi:10.1200/jco.2009.25.0068)

30 Carr LL, Mankoff DA, Goulart BH, Eaton KD, Capell PT, Kell EM, Bauman JE \& Martins RG. Phase II study of daily sunitinib in FDGPET-positive, iodine-refractory differentiated thyroid cancer and metastatic medullary carcinoma of the thyroid with functional imaging correlation. Clinical Cancer Research 201016 5260-5268. (doi:10.1158/1078-0432.ccr-10-0994)

31 Bible KC, Suman VJ, Molina JR, Smallridge RC, Maples WJ, Menefee ME, Rubin J, Karlin N, Sideras K \& Morris JC 3rd et al. A multicenter phase 2 trial of pazopanib in metastatic and progressive medullary thyroid carcinoma: MC057H. Journal of Clinical Endocrinology and Metabolism 201499 1687-1693. (doi:10.1210/ jc.2013-3713)

32 Carlomagno F, Guida T, Anaganti S, Vecchio G, Fusco A, Ryan AJ, Billaud M \& Santoro M. Disease associated mutations at valine 804 in the RET receptor tyrosine kinase confer resistance to selective kinase inhibitors. Oncogene 200423 6056-6063. (doi:10.1038/ sj.onc.1207810)

33 Carlomagno F, Guida T, Anaganti S, Provitera L, Kjaer S, McDonald NQ, Ryan AJ \& Santoro M. Identification of tyrosine 806 as a molecular determinant of RET kinase sensitivity to ZD6474. Endocrine-Related Cancer 200916 233-241. (doi:10.1677/ERC-08-0213)

Received 20 March 2016

Revised version received 1 June 2016

Accepted 8 June 2016 